\title{
An Automated Threshold Selection using Wavelet Based PSO for Image Compression
}

\author{
P.V. Kranthi Kumar ${ }^{1}$, M.S.R Naidu ${ }^{2}$ \\ I(PG Student[DECS], Dept. of ECE, Aditya Institute of Technology and Management, Tekkali, A.P,,India) \\ ${ }^{2}$ (Assoc. Professor, Dept. of ECE, Aditya Institute of Technology and Management, Tekkali, A.P, India)
}

\begin{abstract}
Image Compression addresses the problem of reducing the amount of data required to represent the digital image. Compression is achieved by the removal of one or more of three basic data redundancies known as coding redundancy, Inter pixel redundancy and psycho visual redundancy. Recent research in transformbased image compression has focused on the wavelet transform due to its superior performance over other transforms. In the recent survey, image is subjected to wavelet decomposition to obtain wavelet coefficients and then applied Hard or Soft thresholds for neglecting certain wavelet coefficients by manually selecting global and local threshold values. Here, the manual threshold selection becomes very difficult because it depends on the type of image and its statistical properties like mean and standard deviation. In this paper we proposed an automated threshold selection scheme using wavelet based Particle Swarm Optimization which maintains trade of between peak signal to noise ratio (PSNR) and compression ratio (CR). The above work is implemented using MATLAB 2009.
\end{abstract}

Keywords: CR, Image compression, PSNR, PSO, soft threshold, wavelet decomposition,

\section{Introduction}

With the growth of multimedia technology, the demand for digital information increases drastically. A huge amount of data is produced when a 2 Dimension light intensity function is sampled and quantized. Reduction in file size affords us the advantage of transferring, downloading from the web and storing in hard disk in lesser time period than required. Still images are used in applications like medical imaginary, satellite images, facsimile transmission (FAX) and video conferencing. Image compression addresses the problem of reducing the amount of data required to represent the digital image. Compression is achieved by the removal of one or more of three basic data redundancies: (1) Coding redundancy, which is present when we use more code symbols to represent an image than absolutely necessary. 2) Interpixel redundancy, which results from correlations between the pixels of an image. (3) Psycho visual redundancy which is due to data that is ignored by the human visual system (HVS) information. Image transform coding is the most popular method used in image coding applications. Transform coding is a mathematical operator that converts a large set of highly correlated pixels into a smaller set of uncorrelated coefficients. Transform is a very useful tool in image compression. The spatial redundancies in time domain can be minimized when we transform the image to frequency domain and represented by a few transform coefficients. The purpose of transform coding is to decompose the correlated signal samples into a set of uncorrelated transform coefficients, such that the energy is concentrated into as few coefficients as possible. Over the years, a variety of linear transforms have been developed which include Discrete Fourier Transform (DFT), Discrete Cosine Transform (DCT), Discrete Wavelet Transform (DWT) and many more [1], each with its own advantages and disadvantages. Image compressions using the DCT have already been reported and sufficient literatures are available on this. The JPEG is a glossy compression scheme, which employs the DCT as a tool and used mainly in digital cameras for compression of images. The JPEG standard has been around since the late 1980's and has been an effective first solution to the standardization of image compression. Although JPEG has some very useful strategies for DCT quantization and compression, it was only developed for low compressions. The $8 \times 8$ DCT block size was chosen for speed not for performance. Based on the application one may go for either block DCT or global DCT to process an image to achieve compression.

\section{Wavelet Theory}

The wavelet transform (WT) has gained wide spread acceptance in signal processing and image compression, because of its inherent multi-resolution nature, wavelet coding schemes are especially suitable for applications where scalability and tolerable degradation are important. Recently the JPEG committee has released its new image coding standard, JPEG 2000, which has been based upon DWT. Wavelets are functions defined over a finite interval and having an average value of zero. The basic idea of the wavelet transform is to represent any arbitrary function $f(t)$ as a superposition of a set of such wavelets or basis functions. These 
basis functions are obtained from a single prototype wavelet called the mother wavelet, by dilations or contractions (scaling) and translations (shifts). The Discrete Wavelet Transform of a finite length signal $x(n)$ having $\mathrm{N}$ components, for example, is expressed by an $\mathrm{N} \times \mathrm{N}$ matrix. In many applications wavelet based schemes also referred as sub band coding [2] outperform other coding schemes like the one based on DCT. In wavelet domain, there is no need to do block processing the input image because its basis functions have variable length, wavelet coding schemes at higher compression avoid blocking artifacts which exists in case of block DCT. Wavelet based coding is more robust under transmission and decoding errors, and also facilitates progressive transmission of images which will be useful in internet browsing to effectively use channel band width. In addition, they are better matched to the human visual system (HVS) characteristics. Any signal in continuous time domain can be represented in terms of wavelet basis function as

$x(t)=\sum_{k \in Z} c_{0 k} \varphi_{0 k}(t)+\sum_{j=0}^{\infty} \sum_{k \in Z} d_{j k} \psi_{j k}(t)$

Here $Z$ represents the set of integers and $j$ and $k$ are the scaling and translation parameters respectively. $\left\{\varphi_{j k}(t)=2^{j / 2} \varphi\left(2^{j} t-k\right)\right\}_{k \in Z}$ is an orthonormal basis derived from the scaling function $\varphi(t)$ for the subspace $V_{j} \subset V_{j+1}$, and $\left\{\psi_{j k}(t)=2^{j / 2} \psi\left(2^{j} t-k\right)\right\}_{k \in Z}$ is wavelet functions constitute an orthonormal basis for the subspace $W_{j}=V_{j+1}-V_{j}$. These subspaces define a multi-resolution analysis on $L^{2}(R)$ if the following additional properties are to be satisfied like, $\cap V_{j}=\{0\}, \cup V_{j}$ is dense in $L^{2}(R)$ and a function $s(t)$ is in $V_{j}$ if and only if $s(2 t)$ is in $V_{j+1}$.We can construct the scaling function coefficients $\left(c_{j k}\right)$ and wavelet coefficients $\left(d_{j k}\right)$ by using the relations which are expressed as follows:

$c_{j k}=\int x(t) \varphi_{j k}(t) d t=<x(t), \varphi_{j k}(t)>$
$d_{j k}=\int x(t) \psi_{j k}(t) d t=<x(t), \psi_{j k}(t)>$

\section{Discrete Wavelet Transform (DWT) [5]}

The output of discrete wavelet coefficients of a function being expanded is a sequence of numbers like samples of a continuous function and the resulting transform is called the discrete wavelet transform (DWT). The DWT transform pair is given below

$$
\begin{aligned}
& W_{\varphi}\left(j_{0}, k\right)=\frac{1}{\sqrt{M}} \sum_{x} f(x) \varphi_{j 0, k}(x) \\
& W_{\psi}(j, k)=\frac{1}{\sqrt{M}} \sum_{x} f(x) \psi_{j, k}(x)
\end{aligned}
$$

For $j \geq j_{0}$ and $f(x)=\frac{1}{\sqrt{M}} W_{\varphi}(j 0, k) \varphi_{j 0, k}+\frac{1}{\sqrt{M}} \sum_{j=j 0}^{\infty} \sum_{k} W_{\psi}(j, k) \psi_{j, k}(x)$

Here, $f(x), \varphi_{j 0, k}(x), \psi_{j, k}(x)$ are functions of the discrete variables $x=0,1,2,3, \ldots M-1$. For example, $f(x)=f\left(x_{0}+x \Delta x\right)$ for some $x_{0}, \Delta x$ and $x=0,1,2,3, \ldots M-1$. Normally, we let $j 0=0$ and select $M$ to be a power of 2 so that the summations are performed over $x=0,1,2,3, \ldots M-1, j=0,1,2,3, \ldots J-1$ and $K=0,1,2,3, \ldots 2^{j}-1$. The DWT is identical to a hierarchical sub band system where the sub bands are logarithmically spaced in frequency and represent octave decomposition. The one dimensional filter in (4), (5) can be used as two dimensional separable filters for the processing of images. A separable filter are first applied vertical dimension and then horizontal dimension and then down sampling is performed in two stages once before the second filtering operation to reduce the overall number of computations which is shown in Fig. 1(a). The resulting filtered output denoted LL1, LH1, HL1 and HH1 represent the finest scale wavelet coefficients and LL1 corresponds to coarse level of wavelet coefficients. To obtain the next level of wavelet coefficients, the sub band LL1 alone decomposed into LL2, HL2, LH2, and HH2. This results in two level wavelet decomposition as shown in Fig. 1(b). 


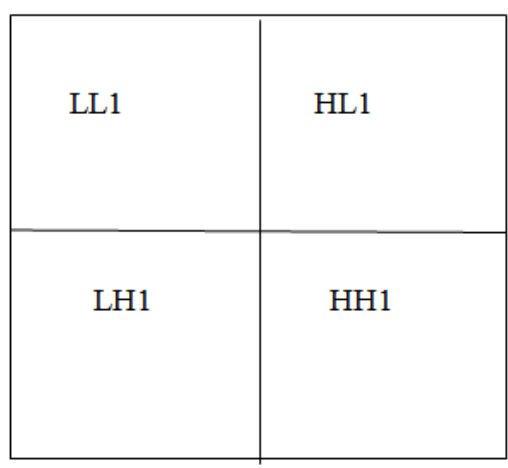

(a) One Level

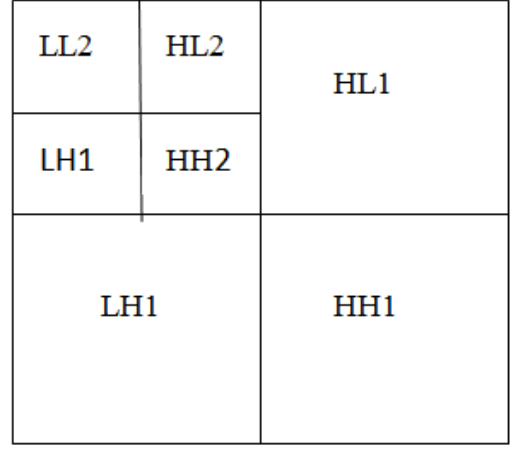

(b) Two -Level

Fig. 1 Image decomposition

Wavelet Transform segregates the information present in the image into approximate and detail signals which are shown in (6). This wavelet analysis depends on the type of the wavelet used, its level of decomposition and threshold value. The approximation signals display pixel values of image and detail signal displays the horizontal, vertical, and diagonal details of an image. Threshold depends on the information which we obtained from the detail signals. Higher compression ratio is the main goal of image compression; higher compression ratio can be also obtained through the selection of threshold values. If the information obtained from detail signals are very small then it is set zero. Higher compression can be achieved when the number of zeros is greater. The image is said to be loss less if the energy retained is $100 \%$ then the compression is known as 'lossless compression'. This occurs when the threshold value is set to zero, meaning that the detail has not been changed. If any values are changed then energy will be lost and this is known as 'lossy' compression. The basic block diagram of wavelet based image compression system is shown in Fig.2. Edges tend to be represented by wavelet coefficients at the finer scales (HL1, LH1, and HH1). Discarding these coefficients would result in a natural filtering which gives the compression [7].

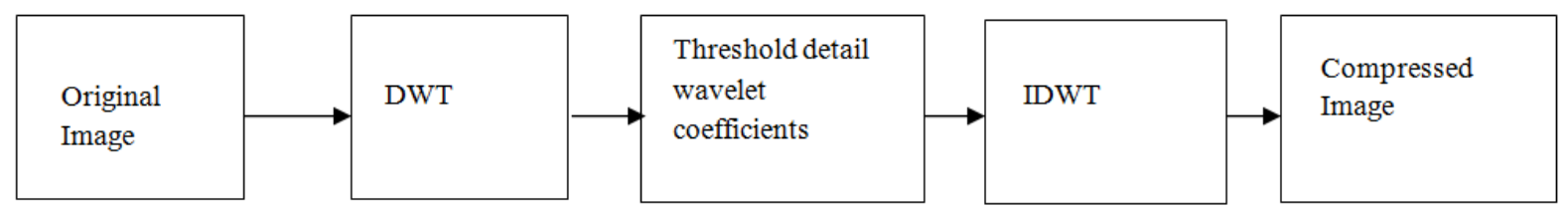

Fig. 2 Wavelet based compression system

Threholding modifies coefficients as per the selected threshold value. Some of the thresholding methods are: 1.Hard thresholding, 2. Soft thresholding. Soft thresholding leads to less severe distortion of the object of the interest than hard thresholding. Thus, to implement a soft and threshold of the DWT coefficients for a particular wavelet band, the coefficients of that band should be thresholded as given in the following.

$$
\begin{aligned}
d_{i k}^{\text {soft }}=\left(\begin{array}{lr}
\operatorname{sign}\left(d_{i k}\right)\left(\left|d_{i k}-\lambda\right|\right) & \text { for }\left|d_{i k}\right|>T \\
0 & \text { for } d_{i k} \leq T
\end{array}\right) \\
d_{i k}^{\text {Hardt }}=\left(\begin{array}{lr}
d_{i k} & \text { for }\left|d_{i k}\right|>T \\
0 & \text { for } d_{i k} \leq T
\end{array}\right)
\end{aligned}
$$

(8)

Where $d_{i k}$ is the wavelet coefficient and $T$ is the threshold value obtained from PSO algorithm [3].

\section{Particle Swarm Optimization}

Computational Intelligence is a successor of artificial intelligence and relies on heuristic algorithms such as neural networks (NN) and evolutionary computation. Algorithms formed on the basis of evolution of biological life in the natural world to solve several computational issues are termed as evolutionary computation. These algorithms are based on the principles of natural evolution. The main aim of research in optimization is to design the most suitable and efficient algorithms for a given optimization task. Recently the interest of engineers is increasing rapidly since the resulting swarm intelligence is applicable in optimization problems in various fields like communication systems, robotics, electrical power systems, image processing, military applications and many more. Meta heuristic algorithms for engineering opti-mization include genetic 
algorithms (GA), simulated annealing (SA), variants of particle swarm optimization (PSO), ant colony algorithm, bee algorithm, harmony search (HS), firefly algorithm (FA), and many others. Particle swarm optimization (PSO) was developed by Kennedy and Eberhart in 1995, based on swarm behavior such as fish and bird schooling in nature. Many algorithms such as ant colony algorithms and virtual ant algorithms use the behavior of the so-called swarm intelligence. Particle swarm optimization may have some similarities with genetic algorithms and ant algorithms, but it is much simpler because it does not use mutation/crossover operators or pheromone. Instead, it uses the real-number randomness and the global communication among the swarm particles. In this sense, it is also easier to implement as there is no encoding or decoding of the parameters into binary strings as those in genetic algorithms which can also use real-number strings. This algorithm searches the space of an objective function by adjusting the trajectories of individual agents, called particles, as these trajectories form piecewise paths in a quasi-stochastic manner. The movement of a swarming particle consists of two major components: a stochastic component and a deterministic component. Let $x_{i}$ and $v_{i}$ be the position vector and velocity for particle $i$, respectively. The new velocity vector is determined by the following formula

$$
v_{i}^{t+1}=w v_{i}^{t}+c_{1} \varepsilon_{1}\left(x_{i}^{*}-x_{i}^{t}\right)+c_{2} \varepsilon_{2}\left(g^{*}-x_{i}^{t}\right)
$$

Here $w$ is the inertia weight, $\varepsilon_{1}$ and $\varepsilon_{2}$ are random numbers usually chosen between $[0,1] . c_{1}$ is a positive constant, called as coefficient of the self-recognition component, $c_{2}$ is a positive constant, called as coefficient of the social component and the choice of value is $c_{1}=c_{2}=2$ generally referred to as learning factors. The initial locations of all particles should distribute relatively uniformly so that they can sample over most regions, which is especially important for multimodal problems. The initial velocity of a particle can be taken as zero. The new position can then be updated by

$x_{i}^{t+1}=x_{i}^{t}+v_{i}^{t+1}$

Algorithm of PSO: Pseudo code of the PSO algorithm is shown below [6]

Objective Function $f(x), x=\left(x_{1}, \ldots ., x_{p}\right)^{T}$

Initialize locations $x_{i}$ and velocity $v_{i}$ of $n$ particles

Find $g^{*}$ from $\min \left\{f\left(x_{1}\right), \ldots, f\left(x_{n}\right)\right\}($ at $t=0)$

While (criterion)

$t=t+1 \quad$ (Pseudo time or iteration counter)

for loop over all $n$ particles and all $p$ dimensions

Generate new velocity $v_{i}^{t+1}$ using equation ()

Calculate new locations $x_{i}^{t+1}=x_{i}^{t}+v_{i}^{t+1}$

Evaluate objective functions at new locations $x_{i}^{t+1}$

Find the current best for each particle $x_{i}^{*}$

end for

Find the current global best $g^{*}$

end while

output the final results $x_{i}^{*}$ and $g^{*}$

Fig. 3 Pseudo code for particle swarm optimization

\section{Experimental Results And Tables}

Two color images, "Lena" and "House", are used as benchmarks to evaluate the performance of the setup using wavelet based PSO approach. The two images, $256 * 256$ in size are taken for the experiment. The performance of the developed system is analyzed using 'haar' wavelet with one level decomposition. The dimension for the problem is considered as ' 1 ' and the population is considered as $20, w=0.6 \quad c_{1}=2$ and $c_{2}=2$. The setup has been implemented for 1 ) If the input is compression ratio then PSO has to find what will be the threshold value which will satisfy the given input compression ratio 2) If the input is PSNR then PSO has to find what will be threshold value which will satisfy the given input PSNR.

4.1). Threshold selection for the given compression ratio (CR) 
The fitness/Objective function defined as

current fitness value $=\frac{\left(3 * M M * M M-W O_{-} T H\right)}{\left(3 * M M * M M-W_{-} T H\right)}-C R_{-} I N P U T$

Where "MM" represents size of the wave let coefficients in any of the wavelet sub band( LL1 or HL1 or LH1 or HH1), WO_TH and W_TH are number of wavelet coefficients having zero value in the original image before soft threholding and after soft threholding respectively and CR_INPUT is the required compression ratio given by the user. If sign of current fitness value of particles in the above expression is - 1 then current position of the particle re initialize and current fitness value is assigned to maximum value 100. Otherwise the loop will be continued to get proper threshold which minimizes the above expression value for the given compression ratio.

4.2). Threshold selection for the given Peak Signal to Noise Ratio (PSNR)

The Objective function defined as

current_fitness_value $=P S N R \_A C T-P S N R \_R E Q$

Where PSNR_ACT is the PSNR value after soft thresholding and PSNR_REQ is the required PSNR value given by the user. From the above, a suitable threshold value will be obtained using PSO algorithm for the given PSNR. If sign of current fitness value of particles in the above expression is -1 then current position of the particle re initialize and current fitness value is assigned to maximum value 100. Otherwise the loop will be continued to get proper threshold which minimizes the above expression value for the given PSNR.

The metrics used to calculate the quality of the image are Compression Ratio (CR), Peak to Signal Noise Ratio (PSNR) and Mean Square Error (MSE [4]).
A. Compression Ratio $=\frac{\text { Ratio of number of zeros of current decompositonlevel }}{\text { number of coefficien ts }}$
B. $\quad P S N R=10 \log _{10}\left(255^{2} / M S E\right)$
C. $M S E=\frac{1}{M N} \sum_{x=0}^{M-1} \sum_{y=0}^{N-1}\left[f(x, y)-f^{\prime}(x, y)\right]^{2}$

where $f(x, y)$ and $f^{\prime}(x, y)$ are original image and threshold images of size $M x N$ respectively.

Table 1: Experiment results threshold value and PSNR for the given compression ratio on "Lena" image.

\begin{tabular}{|l|l|l|l|l|}
\hline S.No & $\begin{array}{l}\text { Input Compression Ratio } \\
\text { (CR in \%) }\end{array}$ & $\begin{array}{l}\text { Threshold value obtained } \\
\text { using PSO }\end{array}$ & $\begin{array}{l}\text { Peak to Signal Noise } \\
\text { Ratio in dB }\end{array}$ & $\begin{array}{l}\text { Actual compression ratio } \\
\text { obtained in\% }\end{array}$ \\
\hline 1 & 5 & 9.43 & 35.69 & 5.22 \\
\hline 2 & 10 & 16.88 & 32.83 & 10.29 \\
\hline 3 & 15 & 22.39 & 31.52 & 15.14 \\
\hline 4 & 20 & 27.10 & 30.73 & 20.62 \\
\hline 5 & 25 & 30.56 & 30.33 & 25.15 \\
\hline 6 & 30 & 33.99 & 30.00 & 30.26 \\
\hline 7 & 40 & 38.99 & 29.57 & 40.80 \\
\hline 8 & 50 & 42.28 & 29.30 & 50.96 \\
\hline
\end{tabular}

Table 2: Experiment results threshold value and PSNR for the given compression ratio on "House" image.

\begin{tabular}{|l|l|l|l|l|}
\hline S.No & $\begin{array}{l}\text { Input Compression Ratio } \\
\text { (CR in \%) }\end{array}$ & $\begin{array}{l}\text { Threshold value obtained } \\
\text { using PSO }\end{array}$ & $\begin{array}{l}\text { Peak to Signal Noise } \\
\text { Ratio in dB }\end{array}$ & $\begin{array}{l}\text { Actual compression ratio } \\
\text { obtained in \% }\end{array}$ \\
\hline 1 & 5 & 6.86 & 39.26 & 5.33 \\
\hline 2 & 10 & 11.88 & 36.53 & 10.34 \\
\hline 3 & 15 & 15.50 & 35.30 & 15.33 \\
\hline 4 & 20 & 19.88 & 34.38 & 20.37 \\
\hline 5 & 25 & 23.60 & 33.68 & 25.31 \\
\hline 6 & 30 & 27.99 & 33.07 & 30.67 \\
\hline 7 & 40 & 33.99 & 32.35 & 40.72 \\
\hline 8 & 50 & 38.27 & 31.88 & 50.01 \\
\hline
\end{tabular}

Table 3: Experiment results threshold value and compression ratio (CR) for the given PSNR on "Lena" image.

\begin{tabular}{|l|l|l|l|l|}
\hline S.No & Input PSNR in dB & $\begin{array}{l}\text { Threshold value obtained } \\
\text { using PSO }\end{array}$ & $\begin{array}{l}\text { Compression ratio (CR in } \\
\%)\end{array}$ & $\begin{array}{l}\text { Actual PSNR obtained in } \\
\text { dB }\end{array}$ \\
\hline 1 & 30 & 33.73 & 30.26 & 30.01 \\
\hline 2 & 32 & 20.10 & 13.27 & 31.94 \\
\hline 3 & 34 & 13.06 & 7.67 & 33.87 \\
\hline 4 & 36 & 8.99 & 4.93 & 36.20 \\
\hline 5 & 38 & 6.37 & 3.69 & 37.90 \\
\hline
\end{tabular}


Table 4: Experiment results threshold value and compression ratio (CR) for the given PSNR on "House" image.

\begin{tabular}{|l|l|l|l|l|}
\hline S.No & Input PSNR in dB & $\begin{array}{l}\text { Threshold value obtained } \\
\text { using PSO }\end{array}$ & $\begin{array}{l}\text { Compression ratio(CR in } \\
\%)\end{array}$ & $\begin{array}{l}\text { Actual PSNR obtained in } \\
\text { dB }\end{array}$ \\
\hline 1 & 32 & 37.23 & 47.59 & 31.96 \\
\hline 2 & 34 & 21.74 & 22.85 & 34.02 \\
\hline 3 & 36 & 13.23 & 12.11 & 35.91 \\
\hline 4 & 38 & 8.54 & 7.19 & 37.97 \\
\hline 5 & 39 & 6.86 & 5.33 & 39.26 \\
\hline
\end{tabular}

\section{Conclusion}

From the above results we can conclude that an automated system can be developed to find threshold value for the given input compression ratio or PSNR. The system is quite useful to know the compression ratio and PSNR values before the transmission of the image in the channel. Based on our requirement we can go for an automated threshold selection system. This paper can be extended by using different wavelets and also using 2 level and 3 level wavelet decompositions. This paper can also be extended by using different variants of PSO and also meta heuristic algorithms like ant colony algorithm, bee algorithm, harmony search (HS), firefly algorithm (FA).

\section{Journal Papers:}

\section{References}

[1] Sindhu M., Rajkamal R, Image and Its Compression Techniques, International Journal of Recent Trends in Engineering, 2(4), 2009, 71-75

[2] S. G. Mallat, A Theory for Multiresolution Signal Decomposition: The Wavelet Representation, IEEE Trans. on Pattern Analysis and Machine Intelligence, 11(7), 1989, 674-693

[3] Sonja Grgic, Mislav Grgic, and Branka Zovko-Cihlar, Performance Analysis of Image Compression using Wavelets, IEEE Trans. On industrial Electronics, 48(3), 2001

[4] Iman Elyasi and Sadesh Zarmehi, Elimination Noise by Adaptive Wavelet Threshold, world academy of science, engineering and technology, 56, 2009.

Books:

[5] Gonzalez, R. C. and Woods, R. E. and Eddins, S. L., Digital Image Processing Using MATLAB (Prentice Hall, 2004)

[6] S.Sumathi and Surekha P, Computational Intelligence Paradigms Theory and Applications using MATLAB ( CRC Press, Taylor \& Fransis Group, Boca Raton, London, New York)

\section{Proceeding Papers:}

[7] J.Abirami, K.Narashiman, S.SivaSankari and S.Ramya, Performance Analysis of Image Compression using Wavelet Thresholding, Proc. 2013 IEEE Conference on Information and Communication Technologies (ICT 2013) 